\title{
Promoting inclusivity by ensuring that all patients with mental health issues are offered research opportunities in the NHS
}

Catherine Henshall ${ }^{1 \& 2}$ http://orcid.org/0000-0001-5659-3296, Helen Jones ${ }^{1}$, Tanya Smith ${ }^{1}$, Andrea Cipriani ${ }^{1 \& 3}$ http://orcid.org/0000-0001-5179-8321

$\left({ }^{1}\right.$ Research and Development Department, Oxford Health NHS Foundation Trust, Oxford, UK; ${ }^{2}$ Oxford School of Nursing and Midwifery, Faculty of Health and Life Sciences, Oxford Brookes University, Oxford, UK; ${ }^{3}$ Department of Psychiatry, University of Oxford, Oxford, UK)

Research-active clinical services have lower mortality rates and produce higher quality care outcomes, however, recruiting participants to clinical research in the National Health System (NHS) remains challenging. ${ }^{1}$ A recent study, assessing the feasibility of clinical staff electronically documenting patient consent to discuss research participation, indicated very low patient uptake, limiting its effectiveness as a strategy for improving access to research. ${ }^{2} \mathrm{~A}$ follow-on study comparing this 'opt-in' approach with an 'opt-out' approach, whereby patients are informed about research opportunities unless they indicate otherwise, found that patients and staff favoured an 'opt-out' approach and wanted research to be more accessible.3 Subsequently, in August 2021, Count me In was developed and launched within Oxford Health NHS Foundation Trust adult and older adult mental health services. Count Me In is an opt-out initiative and a 12-month implementation study, aiming to promote inclusivity by enabling greater equity of information provision for marginalised groups (including Black, Asian and minority ethnic groups, older adults, people with physical and mental disabilities, refugees and asylum seekers), rather than relying on clinician-led recruitment. ${ }^{3}$ It was developed in consultation with our Caldicott Guardian and Head of Information Governance to ensure correct handling of patient data and to differentiate the initiative from 'national data opt-out'. ${ }^{4} \mathrm{~A}$ robust communications plan raised awareness of the initiative (https://www. oxfordhealth.nhs.uk/publication/countme-in/). Patient contact preferences and research involvement are documented on the electronic patient record. Preliminary findings illustrate that in just over 3 months, 8824 patients became contactable through Count Me In, a 400\% increase on the number previously contactable through the 'standard' opt-in. Only 120 patients have opted out of contact. Of 234 potentially eligible patients contacted about specific research studies, 46 (19.6\%) consented to participate. Inclusivity across age, gender, ethnicity and diagnostic group is being monitored and early evidence signals positive changes in equity of research access. For instance, the Count Me In cohort now represents patients across 62 of the 70 diagnostic groups represented in the Trust's caseload, in comparison to only 44 groups represented using the standard 'opt-in' approach. A full evaluation at the end of the 12-month implementation phase will highlight trends and changes in research activity, while also allowing for process modifications to be made before the initiative is rolled out across the country. The ultimate aim is to extend Count Me In to as many NHS Trusts as possible, embedding research within routine patient care and promoting inclusivity by ensuring that research opportunities are offered to all patients with mental health issues, regardless of diagnosis or how well known they are to clinicians.

\section{Acknowledgements}

AC is supported by the National Institute for Health Research (NIHR) Oxford Cognitive Health Clinical Research Facility, by an NIHR Research Professorship (grant RP-2017-08-ST2-006), by the NIHR 
Applied Research Collaboration Oxford and Thames Valley at Oxford Health NHS Foundation Trust and by the NIHR Oxford Health Biomedical Research Centre (grant BRC-1215-20005). CH is supported by the NIHR Senior Nurse and Midwife Research Leader Programme.

\section{References}

1 Jones $\mathrm{H}$, Cipriani A. Barriers and incentives to recruitment in mental health clinical trials. Evid Based Ment Health 2019;22:49-50.

2 Walker S, Potts J, Martos L, et al. Consent to discuss participation in research: a pilot study. Evid Based Ment Health 2020;23:77-82.

3 Henshall C, Potts J, Walker S, et al. Informing National health service patients about participation in clinical research: a comparison of opt-in and opt-out approaches across the United Kingdom. Aust $N$ Z J Psychiatry 2021;55:400-8.

4 NHS Digital. National data opt-out. Available: https:// digital.nhs.uk/services/national-data-opt-out [Accessed 23 Dec 2021]. 